\title{
ChemComm
}

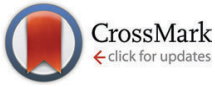

Cite this: Chem. Commun., 2016, 52, 1214

Received 29th October 2015, Accepted 18th November 2015

DOI: $10.1039 / \mathrm{c5cc08992a}$

www.rsc.org/chemcomm

\section{Is it all in the hinge? A kryptoracemate and three of its alternative racemic polymorphs of an aminonitrile $\dagger$}

\author{
R. Laubenstein, ${ }^{a}$ M.-D. Şerb, ${ }^{b}$ U. Englert, ${ }^{c}$ G. Raabe, ${ }^{d}$ T. Braun ${ }^{\star a}$ and B. Braun ${ }^{\star a}$
}

Four polymorphs of 2-(perfluorophenyl)-2-(phenylamino)acetonitrile have been crystallized and structurally analyzed: in addition to three racemic crystals, a rare kryptoracemate has been obtained. The central single bonds allow for conformational flexibility: the kryptoracemate as well as two of the remaining polymorphs contain several independent molecules with different conformation. In contrast to these uncommon packing modes, the fourth phase is unexceptional and crystallizes with a single molecule in the asymmetric unit. Individual crystallization batches may contain several crystal forms concomitantly.

Four alternatives exist for the crystallization of racemic solutions: (a) Ordered racemic structures with both enantiomers in the unit cell represent the most common result. (b) The less frequent second alternative consists in spontaneous resolution; in such conglomerates each crystal is homochiral and hence necessarily belongs to one of the 65 chiral space groups or Sohncke groups. Statistics $^{1-4}$ prove that racemic crystals occur more frequently than conglomerates. This preference for heterochiral crystals has long been associated with better space filling and Wallach's rule $^{5,6}$ even predates X-ray diffraction. Preferred mirror image recognition in crystalline solids is not limited to exact enantiomers but extends to quasiracemates, in which opposite enantiomers of chemically related molecules co-crystallize. ${ }^{7}$ The remaining two

\footnotetext{
${ }^{a}$ Institute of Chemistry, Humboldt University Berlin, Brook-Taylor-Str. 2, 12489 Berlin, Germany. E-mail: beatrice.braun@hu-berlin.de

${ }^{b}$ Faculty of Applied Chemistry and Materials Science,

University Politehnica of Bucharest, Polizu 1, 011061 Bucharest, Romania

${ }^{c}$ Institute of Inorganic Chemistry, RWTH Aachen University, Landoltweg 1, 52074 Aachen, Germany

${ }^{d}$ Institute of Organic Chemistry, RWTH Aachen University, Landoltweg 1 , 52074 Aachen, Germany

$\dagger$ Electronic supplementary information (ESI) available: Photograph of a crystal of krypto-1, table with numerical values for torsion angles in the alternative crystal forms, details concerning synthesis, spectroscopic and analytic characterization of $\mathbf{1}$, crystallization of the alternative crystal forms of $\mathbf{1}$, crystal data, data collection parameters and convergence results for krypto-1, rac-1a, rac-1 b and rac-1c, intermolecular interactions. CCDC 1414258 (krypto-1), 1414259 (rac-1a), 1414260 ( $r a c-1 b)$ and 1414261 (rac-1c). For ESI and crystallographic data in CIF or other electronic format see DOI: 10.1039/c5cc08992a
}

possibilities are only rarely observed: (c) Pseudoracemates ${ }^{8,9}$ are solid solutions of opposite enantiomers; these heterochiral crystals may adopt any space group. (d) Finally, so-called kryptoracemates ${ }^{10,11}$ or false conglomerates ${ }^{9}$ may form; these racemic crystals crystallize in chiral space groups. In their enantiomorphic crystals the opposite enantiomers are "hidden" (such is the meaning of the Greek prefix krypto) as independent molecules in the asymmetric unit. $Z^{\prime}$, the number of molecules per asymmetric unit, is therefore necessarily larger than one when the enantiomers occupy general positions.

Reviews for kryptoracemate crystallization were published by Fábián \& Brock ${ }^{10}$ covering organic and by Bernal \& Watkins ${ }^{11}$ covering metal-organic compounds with a stereogenic metal atom. These authors identified approximately 200 credible kryptoracemates in the Cambridge Structural Database ${ }^{12}$ and mentioned the difficulties in performing an exhaustive search for this class of compounds. In this contribution we communicate the kryptoracemate of 2-(perfluorophenyl)-2-(phenylamino)acetonitrile (1) and the alternative polymorphs of this compound. The chemical constitution of $\mathbf{1}$ as well as the space groups and the content of the asymmetric unit are compiled in Fig. 1.

Prakash et al. ${ }^{13}$ showed that $\alpha$-aminonitriles are accessible in high yields and purity by a three-component Strecker type

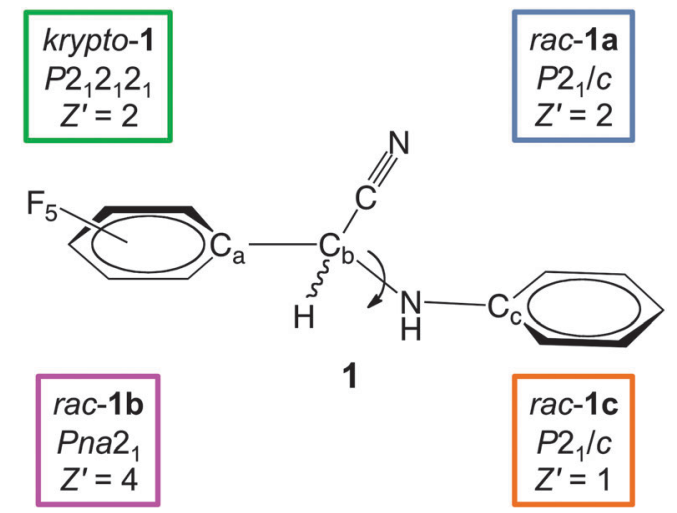

Fig. 1 2-(Perfluorophenyl)-2-(phenylamino)acetonitrile: krypto-1, rac-1a, rac-1b and rac-1c 


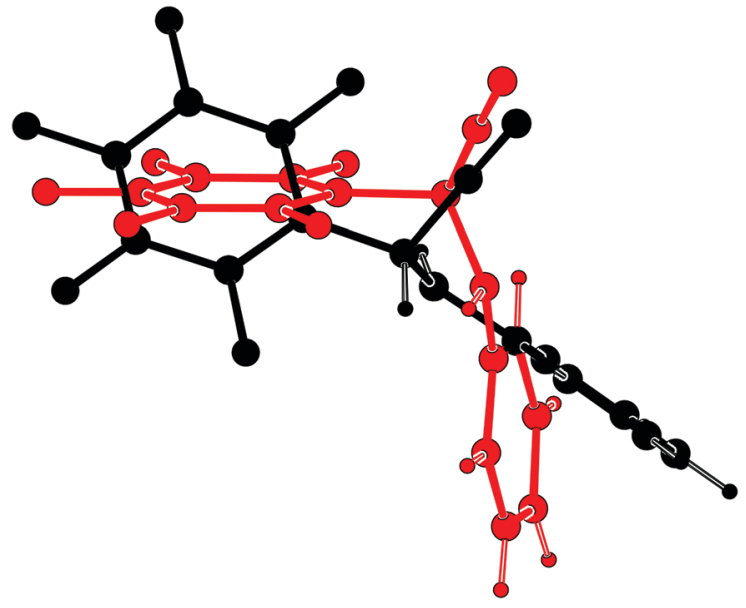

Fig. 2 Overlay (PLATON ${ }^{14}$ ) of the opposite enantiomers in krypto-1

reaction from aldehydes and amines with trimethylsilyl cyanide using gallium triflate as catalyst. In the course of analoguous reactions starting from highly fluorinated benzaldehydes, we came across the racemic compound 2-(perfluorophenyl)-2-(phenylamino)acetonitrile (1). Details concerning synthesis and crystallization have been compiled in the ESI. $\dagger$ The first crystalline appearance of 1 turned out to contain both enantiomers in the asymmetric unit of the chiral space group $P 2_{1} 2_{1} 2_{1}$, thus corresponding to a kryptoracemate (krypto-1). Fig. 2 underlines that the symmetrically independent molecules of opposite chirality adopt very different conformations.

Crystals of krypto-1 are rod-shaped and of good quality; their diffraction pattern extends to high resolution. Very slow isothermal evaporation from hexane at room temperature allowed to grow a single huge elongated crystal (maximum dimension $1.75 \mathrm{~cm}$, weight $13.01 \mathrm{mg}$, see Fig. S1, ESI $\dagger$ ).

After the serendipitous discovery of the unexpected solid krypto-1, we set out in search for potential alternative polymorphs. The next two modifications of $\mathbf{1}$ encountered belong to the most frequent case (a) and correspond to racemic crystals. Crystals of rac-1a are very thin plates in the popular space group $P 2_{1} / c$, those of rac-1b thin needles in the polar space group $P n a 2_{1}$; in either case the quality of the associated intensity data is modest. The solids rac-1a $\left(Z^{\prime}=2\right)$ and $r a c-\mathbf{1 b}\left(Z^{\prime}=4\right)$ both crystallize with more than one independent molecule. Upon closer inspection, they share another peculiarity with krypto-1: all three polymorphs feature symmetrically independent molecules which adopt more or less different conformations with respect to the soft internal degrees of freedom. The single bonds between the perfluorinated and the unsubstituted phenyl rings act as a flexible hinge and allow for different dihedral angles between these aromatic systems. The torsion angle $\mathrm{C}_{\mathrm{a}}-\mathrm{C}_{\mathrm{b}}-\mathrm{N}-\mathrm{C}_{\mathrm{c}}$ (Fig. 1) represents a second soft internal degree of freedom. The symmetrically independent molecules of $\mathbf{1}$ in each of its alternative solid forms krypto-1, rac-1a and rac-1b cover a relevant part of the conformational space thus available. Fig. 3 provides a color-coded synopsis of all individual molecules in the polymorphs of 1, and Fig. 4 allows to visually inspect the variance of the aforementioned degrees of freedom within each modification.
We have attempted to quantify the attribute "more or less different conformations" in our preceding statement: the pairwise least-squares overlay of the nine independent molecules in the different crystal forms of 1 results a matrix ${ }^{15}$ of root mean square (rms) deviations for each of the 36 combinations which allow to address the observed conformations as more or less different. The full matrix is available in the ESI $\dagger$; we here only summarize the main results: (a) one of the molecules in krypto-1, three in rac-1b and the molecule in rac-1c are conformationally similar, with rms values below $0.4 \AA$; these molecules have been marked with an asterisk * in Fig. 3. The two independent molecules in rac-1a and the fourth molecule in $r a c-\mathbf{1 b}$ are conformationally related, albeit to a lower extent. The second molecule in krypto-1 (top row in Fig. 3, middle) stands out completely; it may also readily be identified as the "lone" green dot in the left-hand part of Fig. 4. Up to this point, one might conclude that 1 suffers from a serious packing problem. Its conformational polymorphs krypto-1, rac-1a and rac-10 can then be perceived as the necessarily elaborate answers to this challenge. We recall that structures with an elevated number of independent molecules can be associated with packing conflicts ${ }^{16}$ and that several structures with very high $Z^{\prime 17}$ are based on conformationally flexible constituents. Are the $Z^{\prime}>1$ solids the brute force solution to the packing problem of $\mathbf{1}$ ? Can we hold the central hinge in the molecules of 1 responsible for the manifold and at least in part exotic answers to the packing problem?

We have good reason to doubt the preceding hypothesis. The most simple solution to the packing issue was found by discovering a fourth solid form, rac-1c. It seems to support the often-quoted statement by McCrone ${ }^{18}$ that “... in general, the number of forms known for a given compound is proportional to the time and money spent in research on that compound". During a series of crystallization experiments on $\mathbf{1}$ and after the structural characterization of krypto-1, rac-1a and rac-1b, we detected the unexceptional polymorph rac-1c. It crystallizes in form of apparently hexagonal, well-diffracting plates in the most popular space group $P 2_{1} / c$ with just one molecule in the asymmetric unit. The soft conformational degrees of this only residue fit those of the polymorphs described earlier: this

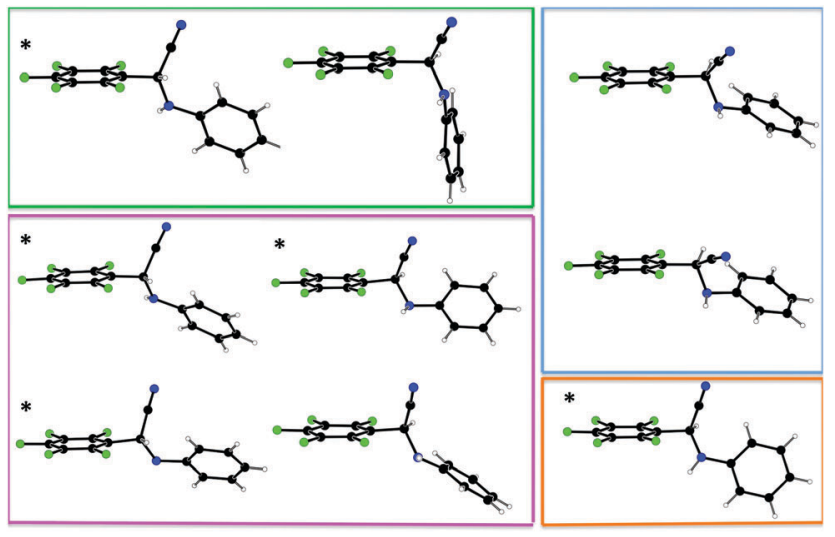

Fig. 3 All independent nine conformations of the molecules in the asymmetric units of krypto-1 (green), rac-1a (blue), rac-1b (magenta) and rac-1c (orange). 


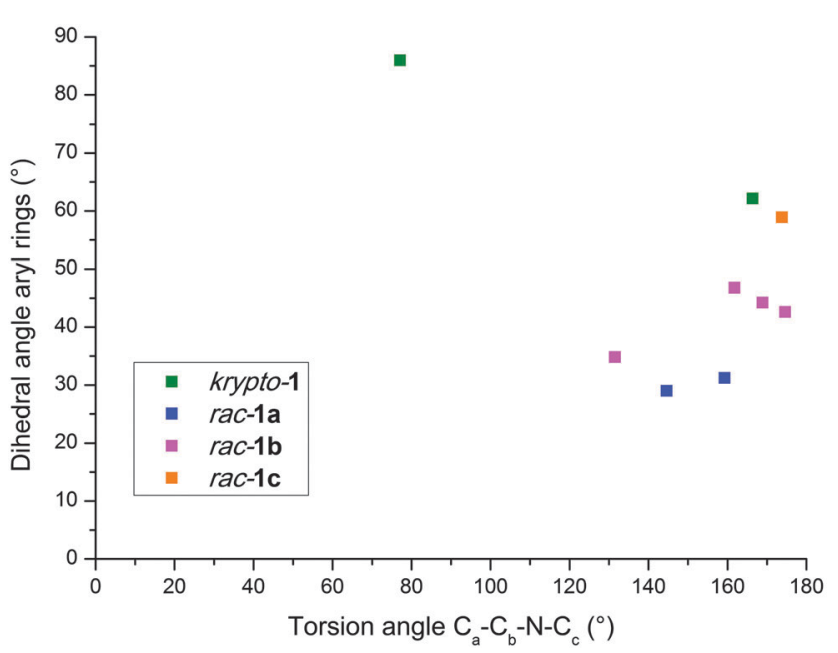

Fig. 4 Mapping of conformational space for the nine conformations; the colors indicate the different polymorphs as in Fig. 3.

underlines that copies of a single molecule can pack sufficiently close and that $Z^{\prime}>1$ is an option rather than a necessary condition for crystallization of $\mathbf{1}$. Table 1 shows that packing in terms of space filling or density is even slightly more efficient for $r a c-1 c$ than for the alternative structures. In other words: the flexible hinge allows for different conformations, but this conformational flexibility is no prerequisite for crystallization.

Intermolecular interactions differ for the alternative phases: in krypto-1, one of the independent moieties has the shortest intermolecular contacts due to $\mathrm{N}-\mathrm{H} \cdots \mathrm{N}$ hydrogen bonds between the hydrogen atom of the secondary amine and a neighboring nitrile $\mathrm{N}$ as acceptor, with $\mathrm{N} \cdots \mathrm{N}$ of 3.102(2) $\mathrm{A}$. This leads to an infinite chain along the $a$ direction (Fig. S2, ESI $\dagger$ ) while the second independent molecule is associated to this chain through $\pi-\pi$ stacking interactions between the electronically different substituted aryl rings of the $(R)$ - and $(S)$ - enantiomers amounting to 3.4975(11) ^ and no slippage (Fig. S2, ESI $\dagger$ ). Overall a one-dimensional structure is generated. Intra-molecular hydrogen bonds between the hydrogen attached to the chiral atom and the nearest fluorine neighboring atoms amount 2.8481(19) $\AA$ and 2.876(2) $\AA$ for the two independent molecules. Between the two independent moieties in the asymmetric unit a weak T-shaped stacking contact (F7- $\mathrm{c}_{\mathrm{g}}$ ) amounting to 3.3293(14) is present. There is also evidence of weak $\mathrm{N} \cdots \mathrm{F}$ possible contacts between the two independent residues. In rac-1a, an infinite one-dimensional chain appear between the hydrogen atoms of the - $\mathrm{NH}$ group and the - $\mathrm{CN}$ groups of different residues with $\mathrm{N}-\mathrm{H} \cdots \mathrm{N}$ bonds which amount to 3.043(5) $\AA$ and 3.087(5) $\AA$. Adjacent chains are running parallel along the $c$ axis (Fig. S5 and S6, ESI†). A short intramolecular

Table 1 Packing properties for the polymorphs of 1

\begin{tabular}{llll}
\hline Polymorph & Space group, $Z^{\prime}$ & Filled space [\%] & Density $\left[\mathrm{g} \mathrm{cm}^{-3}\right]$ \\
\hline krypto-1 & $P 2_{1} 2_{1} 2_{1}, 2$ & 70.1 & 1.583 \\
rac-1a & $P 2_{1} / c, 2$ & 69.3 & 1.569 \\
rac-1b & $P n a 2_{1}, 4$ & 70.9 & 1.603 \\
rac-1c & $P 2_{1} / c, 1$ & 72.8 & 1.651
\end{tabular}

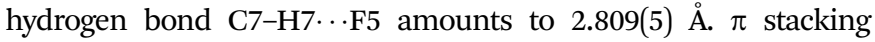
interactions occur between electronically different substituted aryl rings with the shortest distances $\mathrm{c}_{\mathrm{g}}-\mathrm{c}_{\mathrm{g}}$ up to 3.849(3) $\mathrm{A}$ (Fig. S5, ESI†). In $r a c-1 \mathbf{b}$ the two enantiomeric molecules of the same chirality are associated through $\mathrm{N}-\mathrm{H} \cdots \mathrm{N}$ hydrogen bonds amounting to 3.173(11) and 3.209(12) A. Between one residue and its symmetry equivalent $\mathrm{N}-\mathrm{H} \cdots \mathrm{F}$ hydrogen bonds are formed with donoracceptor distance of 2.918(9) ̊̊. Besides, there are $\mathrm{N} \cdots \mathrm{F}$ contacts present giving rise to infinite chains along $b$-axis. The two chains are further associated by weak $\pi-\pi$ stacking interactions with the shortest $c_{g} \cdot c_{g}$ distance of 3.624(7) $\AA$. No pseudosymmetry between the enantiomers of opposite chirality are present. In rac-1c, $\mathrm{N}-\mathrm{H} \cdots \mathrm{N}$ hydrogen bonds of 3.087(2) ̊ form infinite one-dimensional chains in the $c$ direction (Fig. S9 and S10, ESI $\dagger)$. $c_{g} \cdots c_{g}$ distance of 3.8629(10) $\AA$ are rather large. Further details are available in the ESI. $\dagger$

Although $\mathbf{1}$ is well to moderately soluble in the majority of standard laboratory solvents, crystals of all four polymorphs were obtained from slow evaporation of hexane solutions (a solvent in which $\mathbf{1}$ is moderately soluble) at RT (however, in winter and summer our lab temperature varies). Mixture hexane $/ \mathrm{CH}_{2} \mathrm{Cl}_{2}$ also gives rise to crystals of good quality of krypto-1 and rac-1 which can be present in the same batch.

In hindsight, it goes without saying that the sequence of our results plays an important role: if rac-1c had been the first result of a structure determination carried out routinely, only carefully conducted powder diffraction experiments could have saved us from missing the alternative polymorphs.

Equally surprising as the structural manifold among the alternative polymorphs of $\mathbf{1}$ is their harmonious coexistence; we here only mention that the rare kryptoracemate and the most simple form rac-1c can grow concomitantly in the same crystallization batch. This represents the first example of a kryptoracemic structure co-existing with three of its conformational polymorphs, wellordered racemic structures. Future work will be devoted to circular dichroism: the kryptoracemic phase krypto-1 should be associated with a signal in the crystalline state but not in solution.

We thank the Cluster of Excellence "Unifying Concepts in Catalysis" for financial support. Ulli Englert, Gerhard Raabe and Beatrice Braun thank Prof. Gerhard E. Herberich for his stimulating input in many fields and his continuing interest in our work.

\section{Notes and references}

1 F. S. Kipping and W. J. Pope, J. Chem. Soc., Trans., 1897, 71, 989.

2 A. Collet, M. J. Brienne and J. Jacques, Chem. Rev., 1980, 80, 215.

3 C. J. Price, Chem. Eng. Prog., 1997, 93, 37.

4 I. Kuzmenko, I. Weissbuch, E. Gurovich, L. Leiserowitz and M. Lahav, Chirality, 1998, 10, 415.

5 O. Wallach, Liebigs Ann. Chem., 1895, 286, 90.

6 C. P. Brock, W. B. Schweizer and J. D. Dunitz, J. Am. Chem. Soc., 1991, 113, 9811.

7 S. Husebye, Acta Chem. Scand., 1961, 15, 1215; M. S. Hendi, R. E. Davis and K. A. Wheeler, Cryst. Eng., 2000, 3, 209; M. S. Hendi, R. E. Davis, V. M. Lynch and K. A. Wheeler, Cryst. Eng., 2001, 4, 11; U. Englert, R. Haerter, C. Hu, I. Kalf and X. Zheng, Z. Kristallogr., 2000, 215, 627; U. Englert, A. Haering, C. Hu and I. Kalf, Z. Anorg. Allg. Chem., 2002, 628, 1173; S. Reemers and U. Englert, Inorg. Chem. Commun., 2002, 5, 629; I. Kalf, B. Calmuschi and U. Englert, CrystEngComm, 2002, 4, 548; 
B. Calmuschi, M. Alesi and U. Englert, Dalton Trans., 2004, 1852; B. Calmuschi and U. Englert, CrystEngComm, 2005, 7, 171.

8 H. D. Flack, Helv. Chim. Acta, 2003, 86, 905.

9 R. Bishop and M. L. Scudder, Cryst. Growth Des., 2009, 9(6), 2890.

10 L. Fábián and C. Pratt Brock, Acta Crystallogr., Sect. B: Struct. Sci., 2010, 66, 94 and references therein.

11 I. Bernal, J. Cai, S. S. Massoud, S. Watkins and F. Fronczek, J. Coord. Chem., 1996, 38, 165; I. Bernal, J. Cai and J. Myrczek, Acta Chim. Hung., 1995, 132, 451; I. Bernal, F. Somoza and V. Bahn, J. Coord. Chem., 1997, 42, 1; J. Cai, J. Myrczek, H. Chun and I. Bernal, J. Chem. Soc., Dalton Trans., 1998, 4155; I. Bernal and S. Watkins, Acta Crystallogr., Sect. C: Struct. Chem., 2015, 71, 216.

12 WebCSD, 791.568 entries, 9th of Sept. 2015.

13 G. K. S. Prakash, T. Mathew, C. Panja, S. Alconcel, H. Vaghoo, C. Do and G. A. Olah, Proc. Natl. Acad. Sci. U. S. A., 2007, 104, 3703.
14 A. L. Spek, Acta Crystallogr., 2009, D65, 148.

15 Mercury CSD 2.0 - new features for the visualization and investigation of crystal structures; C. F. Macrae, I. J. Bruno, J. A. Chisholm, P. R. Edgington, P. McCabe, E. Pidcock, L. Rodriguez-Monge, R. Taylor, J. van de Streek and P. A. Wood, J. Appl. Crystallogr., 2008, 41, 466.

16 H.-J. Lehmler, L. W. Robertson, S. Parkin and C. P. Brock, Acta Crystallogr., Sect. B: Struct. Sci., 2002, 58, 140.

17 H. Hassaballa, J. W. Steed and P. C. Junk, Chem. Commun., 1998, 577; K. L. Fujdala, A. G. Oliver, F. J. Hollander and T. D. Tilley, Inorg. Chem., 2003, 42, 1140; B. Braun, I. Kalf and U. Englert, Chem. Commun., 2011, 47, 3846.

18 W. C. McCrone, in Physics and Chemistry of the Organic Solid State, ed. D. Fox, M. M. Labes and A. Weissberger, Interscience, New York, 1965, vol. 11, p. 726. 